\title{
Empowering Collaborative Intelligence by the use of User-centered Social Network Aggregation
}

\author{
Xuan Truong Vu, Pierre Morizet-Mahoudeaux, Marie-Hélène Abel \\ UMR CNRS 7253 Heudiasyc \\ Université de Technologie de Compiègne, France \\ Email: $\{x u a n . v u$, pierre.morizet-mahoudeaux, marie-helene.abel $\} @ u t c . f r$
}

\begin{abstract}
Online social networks (OSNs) such as Facebook, Twitter, LinkedIn, Google+, have become extremely popular and ubiquitous today. Users are actively connected to these services for creating and sharing contents and events with others, and, in some cases, this activity takes place in the scope of groups of interests. Therefore, from amongst the morass of data generated every day by users, a part of information may match the interests of certain groups. In practice, members are not all linked to each other via each OSN they are connected to. It is also not realistic to assume that each member can manually explore all others' social profiles to reach the information that may be relevant to their interests. Thus, there is a need for aggregating members' social streams on a single information support to collect relevant information, and, consequently, to promote collaborative knowledge-sharing. However, the disconnected nature of today social websites prevents a straightforward aggregation process. An efficient automated aggregation model is needed. We present, in this paper, the idea of empowering collaborative intelligence by the use of a user-centered approach for OSN aggregation. We illustrate the approach by a first experience to evaluate its impact on users information sharing and enrichment capabilities.
\end{abstract}

Keywords-OSNs, User Profiles Aggregation, Collaborative Intelligence, Knowledge Management

\section{INTRODUCTION}

OSNs have become a very important part of our everyday life [1], from amongst which, social networking services such as Facebook, Twitter, LinkedIn and Google+ are the most well-known examples. These services have hundreds of millions of subscribers around the world. They all allow each user to be a content creator and contributor. However, each OSN has been trying independently to provide users with varied features and user experiences. Facebook, Google+ allow anyone to connect and communicate, Twitter users are able to microblog in real time, and LinkedIn is mainly used in the scope of professional occupations. It is therefore very common that users engage in several OSNs for diverse purposes and interests.

On the other hand, users are also involved in different physical or virtual groups with which they share common interests. They can be professional groups, collective groups, and any other communities. Members are intended to collaboratively contribute and exchange appropriate information inside their respective groups, but actually they spend much more time for doing it on the OSNs to which they subscribed.

In practice, members of a group are not necessarily all connected to the same set of OSNs and even not connected to each other on a OSN. However, a member of a given group may be interested by accessing some part of the information published by the other members in other OSNs. The question is : how can we make it available ? The other related question is : how can we filter only relevant information so as not to overwhelm the user by pointless information. Of course, it is not possible to ask to all the users of a given group to duplicate relevant information they have already published in other OSNs. The proposed solution is to design a system, which allows to aggregate the social streams of members and subsequently to filter only the relevant part of available information. A consequence would be to increase additional information sharing within a group and thus, to promote collaborative knowledge.

We propose an answer to these questions by an approach based on a user-centered social network aggregation to empower collaborative intelligence. User-centered social network aggregation has been firstly introduced in [2] where the authors have proposed : (i) a semantic model, mainly based on the Friend Of A Friend (FOAF) vocabulary and a cross-domain knowledge base such as DBpedia, for merging and modeling users' social data ; (ii) a general architecture for integrating matching information into an organizational knowledge management system. In this paper we present a more achieved and deepen development of this primary approach.

The paper is organized as follows. In the next section, we introduce some of the main characteristics of a collaborative intelligence system in the scope of social aggregated groups. Then we present recent works related to the aggregation of user's data from OSNs. In the fourth section, we introduce our proposed approach of user-centered social network aggregation. The fifth section explains how to match information relevant to the group's interests and how to store it into a collaborative system. In the sixth section we present a case study to evaluate the impact of our approach on users information sharing capabilities an enrichment. Finally we conclude and present our future works. 


\section{Collaborative InTelligence And Social NETWORKS}

In a group, each individual member has only a partial knowledge of what the group is interested in. It is expected that the contribution of each user will be a gain for the entire group since a more complete knowledge will be available and even new knowledge could emerge from the gathering of the contributions. Users thus feel quite natural and willing for sharing their knowledge with others.

On the other hand, we know that social data are very rich and frequently updated. Many advanced systems have used it as a main input to derive a variety of services such as cross-domain recommendation [3], social inter-networking recommendation [4], stock prediction [5]. Regarding a group of users, social data can also be contributive by providing information relevant to the collaboration between members and to the shared interests of the group. Amonsgt others, we have identified four following types of pertinent information

- Users' additional interests : users leave a lot of personal data on their different subscribed OSNs. These data allow to explicitly or implicitly unveil their recent interests, preferences, even expertise [6], [7], which could enrich their partial profile of interests already available in the group.

- New web resources : OSNs are intensively used for publishing and spreading news and Web resources. For example, a significant part of tweets, i.e. short messages published by Twitter users, can be seen as information sharing [8]. Most of them contain URLs referring to web pages, thus allowing to discover new resources matching the interests of the group.

- Emerging topics : by watching recently captured members' additional interests and new Web resources, emerging topics could be identified.

- Possible sub-groups : members of a group can be connected to other members on one or several OSNs. These relationships will give extra indicators for efficiently locating internal sub-groups.

In our approach, we intend to combine the advantages of both collaborative systems and social data. We expect from this combination that the intelligence of a group will be collaboratively empowered by the use of its members' social data. However, an automated process is needed to save users from making manual efforts. We must however keep in mind that one of the key conditions for the success of such an initiative is to provide users with the entire control over the data that they are ready to share. Therefore, we have adopted a user-centered approach. This means that each member will aggregate his/her social profiles by the means of an appropriate tool and decide to share some part of his/her aggregated profile according to his/her preferences and to the common interests of the group.

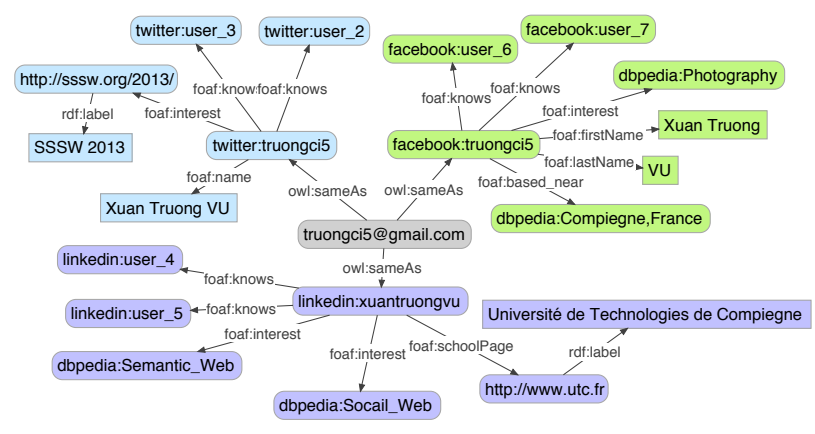

Figure 1. FOAF-based aggregated user profile

In the remaining part of this paper, we will present two main aspects of our approach : the aggregation of users' social profiles and the integration of users' aggregated profile into collaborative systems. The first aspect has been addressed in several related works that we present in the next section.

\section{RELATED WORKS}

With the increasingly growing popularity of OSNs, Social Network Aggregation has become a trending topic which has attracted the attention of both researchers and practitioners. This is a non-trivial problem which can be split into two principal challenges.

The first challenge is how to uniquely identify users across social websites. One of the most notable efforts is the Google Social Graph API ${ }^{1}$. This service, including a matching technique for cross-profiling based on the user homepage, enables an automatic identification. However, it has been no longer available since 2012. Another effort has been introduced in [9] where the authors have proposed an automated matching algorithm which, given an initial set of user attributes like nickname, gender, birthday, city, is able to compute the probable similarity between these initial attributes and crawled data from social websites. The more data are crawled, the more the algorithm is accurate. Nevertheless, due to closeness and authentication requirements of most of popular OSNs, few of users data are publicly available.

The second challenge is how to integrate users' heterogeneous data together. Many commercial solutions, commonly called social network aggregators, such as FriendFeed ${ }^{2}$ and Gathera $^{3}$ allow users to handle their different social accounts via a single location. Zhang et al. [10] have also introduced a personalized OSN aggregator allowing users not only to pull together their social content and activities but also to blend and group friends on different OSNs, and rate the friends and their activities as favorite, neutral or disliked. However,

\footnotetext{
${ }^{1}$ https://developers.google.com/social-graph/

${ }^{2}$ http://friendfeed.com

${ }^{3}$ http://www.gathera.com/
} 
these systems have simply centralized users' data and not really have integrated them.

The most important requirement for integrating users' data is to define a common target data model. Several user models have been developed [11]. A popular solution for social user modeling is using the FOAF vocabulary ${ }^{4}$, one of the most popular lightweight ontologies on the Semantic Web. FOAF makes it possible to represent users' personal information and social relations. Abel et al. [12], in a study of the complementarity of OSNs, have shown that FOAF can be used as a domain specific vocabulary for integrating users' profiles from social websites. However, the study is restricted to a reduced number of public user attributes.

Plumbaum and al. [13] have presented a Social Web User Model intended to fit the needs of social applications. The proposed model encompasses the most frequent user dimensions and attributes available in 17 different social websites. Nevertheless, the final model has not been provided and the social relationship aspect has not been considered. Kapsammer and al. [14] have introduced a comprehensive and extensive reference model for social user profiles, which provides concepts to represent users' information as well as meta information. This work is related to an ongoing research project, and the comprehensibility and computational complexity of the model for domain specific extensions have not been investigated yet.

Recent researches have begun to seek solutions for reusing aggregated users social data, in particular for retrieving users' interests. Shapira and al. [3] have explored the possibility of utilizing declared preferences of music, TV series and movies from users' Facebook profiles for collaborative recommender systems. Noor et al. [15], Abel et al. [7], and Orlandi et al. [6] have modelled users' profiles of interests as structured collections of weighted concepts relevant to the users by using a semantic approach. Their works are based on the analysis of text produced by users on Twitter and Facebook for extracting concepts. Regarding profile representation, they have used DBpedia as a cross-domain knowledge base [16] for concept matching, as well as the Weighted Interests Vocabulary ${ }^{5}$ the Weighting Ontology ${ }^{6}$ for concept weighting.

In our user-centered approach, we explain in the next section that we do not have to tackle the first challenge. The users are asked for directly connecting their social accounts to the system by means of various APIs and authentication protocols. Thus, more users' information can be recovered.

\section{USERS' SOCIAL PROFILES AGGREGATION}

In this section, we first describe our common target user model and then the corresponding aggregation process.

\footnotetext{
${ }^{4}$ http://xmlns.com/foaf/spec/

${ }^{5} \mathrm{http} / / /$ purl.org/ontology/wi/core\#

${ }^{6}$ http://purl.org/ontology/wo/core\#
}

\section{A. A General Social User Model}

In a preceding work we have studied the most frequent properties, which appear to describe the users profiles in the top OSNs (i.e. Facebook, Twitter, LinkedIn, Google+, OpenSocial). From this study, we could build a general user model including six dimensions :

1) The Personal Characteristics includes basic information such as name, city, email, gender, etc.

2) The Friends includes the contacts that the user is connected to.

3) The Interests represents user interests, user preferences and user expertise (e.g. semantic-web, social-network, Facebook, Paris, etc.).

4) The Groups describes the user's memberships.

5) The Studies and Works contains the user's schools and the user's workplaces respectively.

6) The User-created contents includes contents produced by the user (e.g. posts, photos, tags, etc.).

This user model has a twofold function : (i) to indicate to the system which information of the user social profile should be extracted ; (ii) to allow users to choose which information could be shared or not with their group.

The model representation follows the semantic web approach introduced in [6], [12], based on using the FOAF vocabulary and matching the concepts to DBpedia resources. The figure 1 illustrates a simplified view of the aggregated user profile where the user is uniquely identified, by her email, for example. The various social accounts of the same user are represented as separated entities and linked to the user by the owl:sameAs concept. Therefore, each social account has its own attributes, which may be duplicated, or even contain conflicting values. Such a representation allows to preserve in an explicit way the source of anypiece of information (e.g. from Facebook or from Twitter). This feature will also ease users' control over their data and the information sharing.

\section{B. Users' Social Profile Aggregation}

The aggregation process consists of merging the user's various data and of modelling them according to the previous user model. It thus derives several subsequent issues such as entity matching, duplicate or conflict resolution. In addition, when it comes to social data, the user authentication and user data access authorization are also concerned.

We have tackled these issues by a two-step process. The Data Aggregation step is devoted to manage authentication protocols (e.g. OAuth1.0/2.0) and dedicated APIs (e.g. Facebook Graph API, Twitter REST API) for asking users for permissions to access their social profiles and then collecting user data. We have thus developed different aggregators each of which is used for a specific social website.

Once an access is granted, these automated programs will regularly (e.g. everyday) recover new user information relevant to our previous user model. Some social websites like 
Facebook and Twitter provide a very helpful feature called realtime update, to which our aggregators can subscribe to receive certain changes in user data (e.g. feed, friends, likes, etc.) within a couple of minutes of their occurrence.

The User Modelling step attempts to put user data into a unified profile. For this purpose, we have hand-crafted built a set of mapping rules. These rules enable to map straightforwardly gathered information to specific FOAF concepts, for example :

- facebook:User.name = twitter:User.name = foaf:name

- facebook:User.photo $=$ twitter:User.profile_image $=$ foaf.img

- facebook.User.friends $=$ (twitter:User.friends $\cap$ twitter:User.followers) $=$ foaf:knows

Most of properties of Personal Characteristics get a range of literal except current location which will be matched to a related DBpedia place entity. The Studies and Works dimension is described by two properties foaf:schoolHomepage and foaf:workplaceHomepage of which the values are Web page identifiers (i.e. URL). We have also used prefixes such as facebook: or twitter: to indicate internal entities (i.e. users, groups) from corresponding OSNs, for example facebook:truongci5 for representing a Facebook user having "truongci5" as username.

Unlike other kinds of user information, the user 's Interests are less explicit and need additional processing before being merged into the unified profile. In our case, the user's interests are extracted from a list of unstructured texts representing things that users have claimed as belong to their interest. We have used DBpedia Spotlight ${ }^{7}$ and DBpedia keyword search $\mathrm{API}^{8}$ for searching related DBpedia resources from these texts. Matched DBpedia resources will be inserted instead of initial texts. The user's interests can also be extracted from User-created contents, in particular those containing URLs. The same URLs are used to represent the user's interest and described (i.e. rdfs:label) by the title and keywords extracted from referred Web pages.

Every information is kept and translated into triples. In the case of duplicates or conflicts between different social profiles, the user will be later prompted to decide which information should be kept or deleted.

\section{INTEGRATING USERS' AGGREGATED PROFILES INTO COLLABORATIVE INFORMATION SYSTEMS}

For integrating users' social data into collaborative information systems, we have built an extensive system architecture made up of two main parts (figure 2) : (1) the capitalization feature for aggregating users' social data and filtering relevant information ; (2) a collaborative system for management and sharing gathered information. We intend to use an existing collaborative system for (2) on which we will

\footnotetext{
${ }^{7}$ https://github.com/dbpedia-spotlight/dbpedia-spotlight

${ }^{8}$ https://github.com/dbpedia/lookup
}

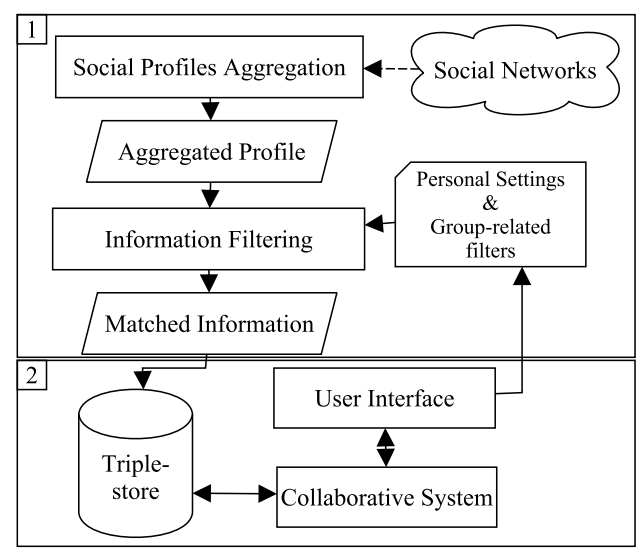

Figure 2. Architecture for integrating users' aggregated profiles into collaborative systems

implement (1) as an extension (i.e. plug-in). This extension is composed of two major modules : the Social Profile Aggregation module, which has been already explained in the previous section, and the Information Filtering module that we describe in detail in this section.

Information Filtering is a crucial step of the data integration process since it must simultaneously address the user privacy and the information matching issues. The former is a very challenging problem taking into account existing privacy laws. We will not address this problem in this paper. Nevertheless, we have implemented light features to comply with user privacy. Before using our system, each user is informed about its objectives and its operations, and is asked to explicitly grant suitable permissions to the extension so that it can access, collect and process their social data. Granted access can be revoked by the users whenever they feel it necessary. The users will also be free to set and modify the following personal settings :

- The social profiles the information of which will be aggregated and filtered (e.g. only Facebook or only Twitter or both),

- The dimension(s) which can be shared (e.g. only Friends and Interests),

- A final review, with the possibility of deselecting undesired information for sharing, before confirmation can be chosen by the user. By default all matched information will be available to other members

For matching and filtering the relevant part to a group's shared interests from its members' aggregated profiles, we have developed different filtering techniques including manual as well as automated methods as below :

- Hashtag Method : a hashtag is a word or a phrase prefixed with the symbol \#. For example, \#WI2013 could stand for the Web Intelligence 2013 conference. Hashtags have become very popular and efficient means for grouping and retrieving messages related to a given 
topic on social websites. The users of our system will be encouraged to use their commonly defined hashtags across OSNs. Gathered contents including such hashtags will be considered pertinent for the group and directly accessible.

- Keyword-based Method : keywords and their synonyms can be also used for matching information. However, the keywords should be specific terms (e.g. "usercentered") or named entities (e.g. "WI2013") to avoid ambiguity and hight recall.

- Ontology-based Method : in comparison to keywords, ontology gives more powerful performance for matching information. Firstly, it creates less ambiguities. Secondly, it is not necessary to list all concepts, named entities in particular, which belong to certain categories. Let's consider an example from DBpedia, in which the Social Networking Services category ${ }^{9}$ is the subject (i.e. dcterms:subject) of a lot of networking services such as dbpedia:Facebook, dbpedia:Twitter, dbpedia:Myspace, dbpedia:Instagram, dbpedia:FOAF_(software), etc. In such a case, only the category will be needed in order to match information related to one of its members.

- Empirical Methods, additional empiric methods can be also used to reduce the number of information extracted from user-created contents. They actually tend to filter out personal messages which are self-describing or addressed to a particular person. For this purpose, they rely on some simple detection patterns such as containing emoticons (e.g. "tired and upset :(") or including other usernames (e.g. "take a look at these photos http://bit.ly/Ywg7p6 @truongci5”).

Only matched information after the Information Filtering process will be stored and shared while other information will be definitively removed.

\section{A STUDY CASE}

In this section, we present our first experience. The main objective was to obtain a real dataset of users and their social data in order to validate the two hypothesis of our proposed approach : (i) users' willingness for information sharing (ii) the usability of collected social data according to the center of interest of a given group.

Profile data have been collected from volunteered employees of the 50A company, specialized in web development and social media, which is supporting the work. We have presented our approach to the employees as well as the objective and the conditions of the experience. They have been informed that the data, gathered during this step of experience, is only used for quantitative studies and will remain totally anonymous. Therefore, thirteen employees have freely and voluntarily participated to the experience. They have different backgrounds (e.g. developers, web masters,

\footnotetext{
${ }^{9}$ dbpedia.org/resource/Category:Social_networking_services
}

project managers, designers and $R \& D$ engineers) but share the same interests for social media, web-based technologies and trending mobile applications.

We have developed a simple application which allows the participants to connect their social profiles and recovers their social data. Each participant is free to choose to connect his/her Facebook profile or Twitter profile. Nine of them have connected both profiles while one has not connected any profile. This is a good indicator that the participants are in majority interested by the proposed idea and willing for sharing their social data with a professional group.

To evaluate the potential of social data, we have programmed the application so that it crawls every day the profiles of the participants and stores new information during 3 weeks (from 20/04/2013 to 10/05/2013). We have obtained data as illustrated in tables I, II and III where total means the number of new items (e.g. interests, contents $=$ tweets $\cap$ facebook posts, contents containing URLs) retrieved from all participants' profiles whereas max, min and avg signifies the biggest, the smallest and the average number of items retrieved from one's social profiles.

In average, during three weeks, a participant has added 9 new subjects of interest, has produced 50 new contents and has shared 35 links either on Facebook or Twitter.

Table I

PARTICIPANTS' NEW INTERESTS

\begin{tabular}{|c|c|c|c|}
\hline Total & Max & Min & Avg \\
\hline 107 & 62 & 0 & 9 \\
\hline
\end{tabular}

Table II

ALL CONTENTS PRODUCED BY PARTICIPANTS

\begin{tabular}{|c|c|c|c|}
\hline Total & Max & Min & Avg \\
\hline 605 & 185 & 1 & 50 \\
\hline
\end{tabular}

Table III

CONTENTS CONTAINING LINKS SHARED BY PARTICIPANTS

\begin{tabular}{|c|c|c|c|}
\hline Total & Max & Min & Avg \\
\hline 422 & 134 & 0 & 35 \\
\hline
\end{tabular}

We have randomly extracted a subset of 100 shared links (i.e. $24 \%$ of all shared links) in order to manually analyze and filter out resources relevant to the three interests (i.e. social media, web-based technologies and trending mobile applications). Subsequently, we have identified 20 candidates (i.e. $20 \%$ of analyzed links) of which 2 are shared twice by two different participants. Here follows some examples of interesting websites.

- http://t.co/B85yeWMOnh : infographic on social medias

- http://t.co/L5cv647B64 : new Facebook Logo

- http://t.co/ZcCMmgqLFa : Twitter-based applications

- http://t.co/SXIbIJCer1 : One Day app by Google

- http://t.co/ibSq5sAUqL : Facebook-powered app, thinglink

- http://t.co/sXxJmoawvX : Wolfram Alpha Personal Analytics for Facebook

- http://t.co/Z76OdX80g1 : social media for business 
We have also investigated all new interest topics which are essentially Facebook pages liked by the participants. A large part of liked pages are related to people, bands, events of either music, cinema or television. Another important part is concerned with brands or products. A few of pages are really matching the social media, web-based technologies and mobile applications topics (e.g. Talenteo, Pipture, Pitomani, Flowtab, Musigram, etc.).

In brief, user-created contents can constitute a potential source of relevant information for the group's center of interests while users' declared likes are mostly related to music, cinema or television.

\section{CONCLUSION AND FUTURE WORK}

In this paper, we have presented a approach for combining the benefits of both collaborative systems and OSNs. A usercentered approach is recommended for enabling the access to users' cross-social-network data and for allowing users to have a better control on their shared information. Therefore, we introduced a general social user model including the main aspects of the users of OSNs and a corresponding social profile aggregation process. We described an extensive system architecture for integrating users' aggregated profiles into collaborative systems. A variety of methods for matching and filtering information were also proposed.

The first experience with a group of real users has proved the two initial hypothesis of our user-centered approach : (i) users' willingness for sharing their social data to their group and (ii) the potential of users' social data. We noticed that users are quite ready for sharing their social data with their respective groups, in particular professional groups and that user-created contents can provide a potential source of relevant information to empower collaborative systems.

On-going work will focus on the implementation of the social extension on an actual collaborative system. To this end, we have planed to use a platform called Memorae (http://www.hds.utc.fr/memorae/). This platform developed at the Université de Technologies de Compiègne, is an ontology-based collaborative environment easing organizational learning and knowledge capitalization. It has been recently improved to integrate and index resources from OSNs [17]. It will therefore allow to extend our experience to bigger groups of real users whose the center of interests might be different from those investigated within our first study case.

\section{ACKNOWLEDGMENT}

Part of this work has been developed in cooperation with the 50A Company (www.50a.fr) which is funding this work.

\section{REFERENCES}

[1] W. Kim, O.-R. Jeong, and S.-W. Lee, "On social web sites," Inf. Syst., vol. 35, no. 2, pp. 215-236, Apr. 2010.
[2] X. T. Vu, M.-H. Abel, and P. Morizet-Mahoudeaux, "An aggregation model of online social networks to contribute to organizational knowledge management," in Proceedings of the 1st international conference on Knowledge Management, Information and Knowledge Systems, Tunisia, 2013.

[3] B. Shapira, L. Rokach, and S. Freilikhman, "Facebook single and cross domain data for recommendation systems," User Modeling and User-Adapted Interaction, vol. 23, no. 2-3, pp. 211-247, 2013.

[4] P. D. Meo, A. Nocera, G. Terracina, and D. Ursino, "Recommendation of similar users, resources and social networks in a social internetworking scenario," Information Sciences, vol. 181, no. 7, pp. 1285 - 1305, 2011.

[5] J. Bollen, H. Mao, and X.-J. Zeng, "Twitter mood predicts the stock market," CoRR, vol. abs/1010.3003, 2010.

[6] F. Orlandi, J. Breslin, and A. Passant, "Aggregated, interoperable and multi-domain user profiles for the social web," in Proceedings of the 8th International Conference on Semantic Systems. New York, NY, USA: ACM, 2012, pp. 41-48.

[7] F. Abel, Q. Gao, G.-J. Houben, and K. Tao, "Analyzing Temporal Dynamics in Twitter Profiles for Personalized Recommendations in the Social Web," in Proceedings of ACM WebSci '11, 3rd International Conference on Web Science, Koblenz, Germany. ACM, Jun. 2011.

[8] M. Naaman, J. Boase, and C.-H. Lai, "Is it really about me?: message content in social awareness streams," in Proceedings of the 2010 ACM conference on Computer supported cooperative work. New York, USA: ACM, 2010, pp. 189-192.

[9] F. Carmagnola, F. Osborne, and I. Torre, "User data distributed on the social web: how to identify users on different social systems and collecting data about them," in Proceedings of the 1st International Workshop on Information Heterogeneity and Fusion in Recommender Systems, ser. HetRec '10. New York, NY, USA: ACM, 2010, pp. 9-15.

[10] J. Zhang, Y. Wang, and J. Vassileva, "Socconnect: A personalized social network aggregator and recommender," Inf. Process. Manage., vol. 49, no. 3, pp. 721-737, May 2013.

[11] F. Carmagnola, F. Cena, and C. Gena, "User model interoperability: a survey," User Modeling and User-Adapted Interaction, vol. 21, no. 3, pp. 285-331, Feb. 2011.

[12] F. Abel, N. Henze, E. Herder, and D. Krause, "Linkage, aggregation, alignment and enrichment of public user profiles with mypes," in Proceedings of the 6th International Conference on Semantic Systems, New York, USA, 2010, pp. 11:1-11:8.

[13] T. Plumbaum and S. Wu, "User Modeling for the Social Semantic Web," 2011, pp. 1-12.

[14] E. Kapsammer, S. Mitsch, B. Pröll, W. Retschitzegger, W. Schwinger, M. Wimmer, M. Wischenbart, and S. Lechner, "Towards a Reference Model for Social User Profiles: Concept \& Implementation," in International Workshop on Personalized Access, Profile Management, and Context Awareness in Databases (PersDB) at VLDB 2011, 2011.

[15] S. Noor and K. Martinez, "Using social data as context for making recommendations: an ontology based approach," in Proceedings of the 1st Workshop on Context, Information and Ontologies, New York, USA, 2009, pp. 7:1-7:8.

[16] P. N. Mendes, M. Jakob, and C. Bizer, "Dbpedia for nlp: A multilingual cross-domain knowledge base," in Proceedings of the Eight International Conference on Language Resources and Evaluation (LREC'12), Istanbul, Turkey, May 2012.

[17] E. Deparis, M.-H. Abel, and J. Mattioli, "Modeling a social collaborative platform with standard ontologies," 2012 Eighth International Conference on Signal Image Technology and Internet Based Systems, vol. 0, pp. 167-173, 2011. 